(NASA-CR-200034) EFFECT OF BAFFLE N96-17147

ON SLOSH REACTION FORCES IN

ROTATING LIQUIO HELIUM SUBJECTED TO A LATERAL IMPULSE IN MICROGRAVITY (Alabama Univ.) $9 \mathrm{p}$

Unclas

G3/34 0092358 


\title{
Effect of baffle on slosh reaction forces in rotating liquid helium subjected to a lateral impulse in microgravity
}

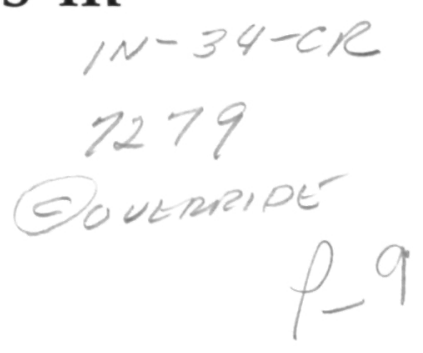

\begin{abstract}
Sloshing dynamics within a partially filled rotating Dewar of superfluid $\mathrm{He}$ II are investigated in response to a lateral impulse. The study investigates several factors, including how the rotating bubble of superfluid He II reacts to the impulse in microgravity, how the amplitudes of slosh reaction forces act on the Dewar with various rotating speeds, how the frequencies of the sloshing modes excited differ in terms of differences in rotating speeds, and how the sloshing dynamics differ with and without a baffle. The numerical computation of sloshing dynamics is based on the non-inertial frame spacecraft-bound co-ordinate. Results of the simulations are illustrated.
\end{abstract} Keywords: helium; sloshing dynamics; mathematical analysis; microgravity; slosh
reaction forces

To carry out scientific experiments, some experimental spacecraft use cryogenic cooling for observation instrumentation and telescopes, and superconducting sensors for gyro read-out, and have to maintain very low temperatures (near absolute zero) for mechanical stability. The approaches to both cooling and control involve the use of He II. In this study, the response of cryogenic systems to sloshing dynamics associated with impulsive oscillations is investigated. The spacecraft uses cooling and boil-off from the liquid helium Dewar (employing the helium as cryogen and propellant) to maintain cooling of instruments, attitude control and drag-free operation of the spacecraft. Potential fluid management problems may arise due to asymmetric distribution of liquid helium and vapour or perturbations at the free surface. Baffle-boards may need to be installed inside the Dewar container to reduce the degree of asymmetry and damp disturbances in the liquid-vapour distribution.

Liquid $\mathrm{He}$ II at a temperature of $1.8 \mathrm{~K}$ is used as the coolant. With its superfluid behaviour, there is a very small temperature gradient in the liquid helium. With the negligibly small temperature dependence of the surface tension and the small temperature gradient along the surface, which lead to Marangoni convection, the equilibrium shape of the free surface is governed by a balance of capillary, centrifugal and gravitational forces. Determination of liquidvapour interface profiles based on computational experiments can uncover details of the flow which cannot be easily visualized or measured experimentally in a microgravity environment.

The instability of the liquid-vapour interface can be induced by the presence of longitudinal and lateral acceler- ations. Thus, slosh waves are excited, producing high and low frequency oscillations in the liquid helium. The sources of the residual accelerations include the effects of the Earth's gravity gradient and $g$-jitter ${ }^{1-3}$. A recent study ${ }^{4}$ suggests that the high frequency accelerations may be unimportant in comparison with the residual motion caused by low frequency accelerations.

The time-dependent behaviour of a partially filled rotating Dewar of fluid in reduced gravity environments was simulated by numerically solving the Navier-Stokes equations subject to initial and boundary conditions ${ }^{5,6}$. At the interface between the liquid and the vapour fluids, both the kinematic surface boundary condition and the interface stress conditions for components tangential and normal to the interface were applied ${ }^{2,3}$. The initial conditions were adopted from the steady state formulations developed by Hung et al. ${ }^{4}$. Some of the steady state formulations of interface shapes were compared with the available experimental results of Leslie ${ }^{5}$ in a free-falling aircraft $(\mathrm{KC}-135)$. The experiments carried out by Mason et al. ${ }^{6}$ showed that classical fluid mechanics theory is applicable to cryogenic helium in large containers with sufficiently large velocities $^{10}$.

At temperatures close to absolute zero, quantum effects begin to be of importance with respect to the properties of the fluids. At a temperature of $2.17 \mathrm{~K}$, liquid helium has a $\lambda$-point (a second-order phase transition); at temperatures below this point, liquid helium (He II) has a number of remarkable properties, the most important of which is superfluidity. This is the property of being able to flow without viscosity in narrow capillaries or gaps. At tempera- 
tures other than zero, He II behaves as if it were a mixture of two different liquids. One of these is a superfluid and moves with zero viscosity along a solid surface. The other is a normal viscous fluid. The two motions occur without any transfer of momentum from one to another for velocities below a critical velocity ${ }^{10-12}$. Considering the components of the normal and superfluid velocities above a critical velocity, the two fluids are coupled through snarling in a complex tangle ${ }^{10-12}$

The key parameter of the critical velocity, to distinguish one-fluid from two-fluid models is a function of fluid temperature and container size. In other words, in considering the dynamic behaviour of He II in a large rotating cylinder, a mixture of the superfluid and the normal fluid, without separation of the two fluids for fluid velocities greater than the critical velocity, is accounted for in the model computation. The density concentration of the superfluid is a function of temperature, which is also true for the surface tension and viscous coefficient for $\mathrm{He}^{\mathrm{II}^{10-15}}{ }^{10}$ In this study, the theory of viscous Newtonian fluids is employed with transport coefficients being a function of temperature.

In this paper, the response of superfluid He II in a Dewar with various rotating speeds to a lateral impulse is studied. To reduce the degree of asymmetry in the liquid-vapour distribution and damp its associated disturbances, a number of baffle boards are installed inside the Dewar. The sloshing dynamics, the modulated liquid-vapour interface disturbances, and the sloshing reaction forces and torques exerted on the Dewar at various rotating speeds with and without the baffle in response to a lateral impulse are also investigated.

\section{Non-inertial frame mathematical formulation of fundamental equations}

Experiments undertaken by Andronikashvili ${ }^{10-12}$ for rotating He II show that it is necessary to exceed a critical velocity for the interaction between the normal and superfluid components to be established throughout the container during rotation ${ }^{10-12}$. For a rotating Dewar with an outer diameter of $1.56 \mathrm{~m}$ and an inner diameter of $0.276 \mathrm{~m}$, the critical velocities needed to assume interaction between the normal and superfluid components are $6.4 \times 10^{-7}$ and $3.6 \times 10^{-6} \mathrm{~m} \mathrm{~s}^{-1}$, respectively ${ }^{10-12}$. With a rotating speed of $0.1 \mathrm{rev} \mathrm{min}^{-1}$, the linear velocities along the outer and inner walls of the rotating Dewar are $8.17 \times 10^{-3}$ and $1.45 \times 10^{-6} \mathrm{~m} \mathrm{~s}^{-1}$, respectively, which are at least several hundred times greater than that of the corresponding critical velocities needed to ensure interaction between the normal and superfluid components of the He II. Based on this illustration, the problem under consideration has special features which warrant the adoption of a viscous Newtonian fluids formulation in this study. These special features are as follows: (a) the fluid velocities are at least several hundred times greater than that of the corresponding critical velocities; (b) these high fluid velocities can produce great enough vortex lines to snarl in a complex tangle to ensure interaction between the normal and superfluid components ${ }^{10-15}$; and (c) this snarling of the vortex lines with a complex tangle between the normal and superfluid components warrants the adoption of the Newtonian fluid model $^{10-15}$.

Consider a closed circular Dewar partially filled with $\mathrm{He}$ II while the rest of the ullage is filled with helium vapour.
The whole fluid system is spinning in the axial direction $z$ of cylindrical co-ordinates $(r, \theta, z)$, with corresponding velocity components $(u, v, w)$. The governing equations for non-inertial frame spacecraft-bound co-ordinates spinning along the $z$-axis have been given in recent studies ${ }^{16-18}$. In other words dynamic forces, such as the gravity gradient, $g$-jitter, angular accelerations, and centrifugal, Coriolis, surface tension and viscous forces, are given explicitly in the mathematical formulations ${ }^{16-18}$. In the computation of the sloshing reaction forces, and the moments, viscous stress and angular momentum acting on the container wall of the spacecraft, one must consider those forces and moments in the inertial frame rather than in the non-inertial frame ${ }^{16-18}$.

For the purpose of solving sloshing dynamic problems of liquid systems in orbital spacecraft under a microgravity environment, one must solve the governing equations ${ }^{17.18}$ accompanied by a set of initial and boundary conditions. A detailed illustration of these initial and boundary conditions concerning the sloshing dynamics of fluid systems in microgravity was precisely given in work by Hung and Pan ${ }^{16,17,19}$. The computational algorithm applicable to cryogenic fluid management under microgravity has also been given earlier ${ }^{17-19}$. A summary of the computational algorithm is illustrated in Figure 1. In this study, in order to show a realistic example, a Dewar with an outer radius of

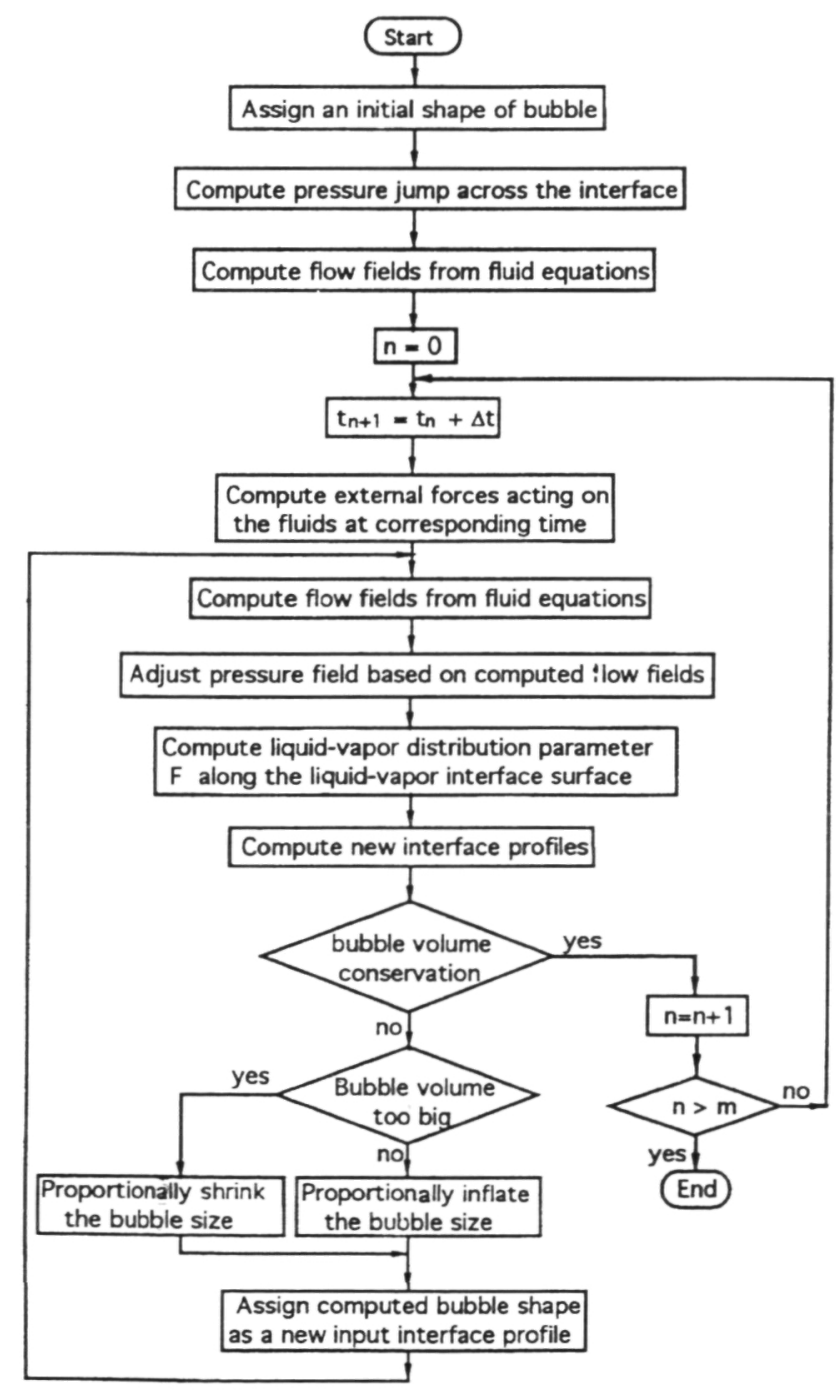

Figure 1 Computation algorithm for sloshing dynamics 
$0.78 \mathrm{~m}$, and an inner radius of $0.138 \mathrm{~m}$, a top and bottom radius of $1.10 \mathrm{~m}$ and a height of $1.62 \mathrm{~m}$ has been used in the numerical simulation. The Dewar tank is $60 \%$ filled with liquid helium and the rest of the ullage is filled with helium vapour. The temperature of liquid helium is $1.8 \mathrm{~K}$. In this study the following data were used: liquid helium density $=145.7 \mathrm{~kg} \mathrm{~m}^{-3}$, helium vapour density $=$ $1.47 \mathrm{~kg} \mathrm{~m}^{-3}$, fluid pressure $=1.6625 \mathrm{~Pa}$, surface tension coefficient at the interface between liquid helium and helium vapour $=0.0353 \mathrm{~N} \mathrm{~m}^{-1}$, liquid helium viscosity coefficient $=9.61 \times 10^{-9} \mathrm{~m}^{2} \mathrm{~s}^{-1}$ and contact angle $=0^{\circ}$. The initial profiles of the liquid-vapour interface for the rotating Dewar are determined from computations based on algorithms developed for the steady state formulation of microgravity fluid management ${ }^{7,20}$.

A staggered grid for the velocity components is used in this computer program. The marker and cell (MAC) method $^{17}$ of studying fluid flows along a free surface is adopted and the volume of fluid (VOF) method is used to solve finite difference equations numerically. The approximate flow velocity is calculated from the explicit approximation of momentum equations based on results from the previous time step. Computations of the pressure and velocity at the new time step are thus carried out by iteratively solving the pressure equation via a conjugate residual technique ${ }^{22-25}$. The configuration of the liquid-vapour interface adjusted by the surface tension effect at the new time step is then finally obtained. The time step during this computation is automatically adjusted through fulfilment of the stability criteria of the computed grid size. The convergence criterion for iteration of the pressure equation is based on the computed velocity at each cell which satisfies the continuity equation with an error of no more than $10^{-5}$ in terms of velocity difference ${ }^{26}$. As for the volume conservation of liquid, a deviation of less than $1 \%$ in volume is always guaranteed before moving to the next time step. Figures $2 a, b$ and $c$ show the three-dimensional configuration of the baffle installation, and the distribution of grid points for the Dewar tank with baffle boards in the radial- axial plane and radial-circumferential plane, respectively, in terms of cylindrical co-ordinates. The baffle-boards are installed with three parallel plates perpendicular to the rotating axis and four radial plates aligned with the rotating axis. The inner radius, outer radius and thickness of the three parallel baffle-boards chosen in this computation are $60,77.48$ and $0.1016 \mathrm{~cm}$, respectively. These three baffles are installed at locations $z_{1}, z_{2}$ and $z_{3}$ of $38.74,80.94$ and $84.4 \mathrm{~cm}$, respectively. Four radial baffle-boards are located $90^{\circ}$ apart with the same inner and outer diameters and thickness as those of the three parallel baffle-boards.

\section{Microgravity sloshing dynamics in response to lateral impulse}

Three examples are given to illustrate the sloshing dynamics of partially filled liquid helium in a Dewar container with rotating speeds of $0.1,0.2$ and 0.3 rev min ${ }^{-1}$ in response to a lateral impulse during the time period of guidance and/or attitude control. In this study, the following form of lateral impulse is assumed:

$\hat{a}=\left[a_{x}, a_{y}, a_{z}\right]=[1,0,0] 10^{-2} g_{0}$ in cartesian co-ordinates $\hat{a}=\left[a_{r}, a_{\theta}, a_{z}\right]=[\cos \theta,-\sin \theta, 0] 10^{-2} g_{0}$ in cylindrical coordinates for $0<t \leq 10^{-2} \mathrm{~s}$

and

$\hat{a}=[0.0 .0]$ for $t>10^{-2} \mathrm{~s}$

where $g_{0}\left(=9.81 \mathrm{~m} \mathrm{~s}^{-2}\right)$ is the Earth's gravitational acceleration.

The time evolution of the liquid-vapour interface without a baffle is first investigated. Figures $3(A) a, b$ and $c$ show three-dimensional bubble deformations with Dewar

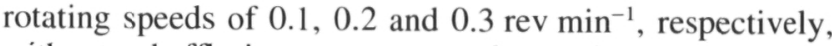
without a baffle in response to a lateral impulse. Figures $4(A) a, b$ and $c$ show the corresponding bubble deformations accompanied by flow profiles with Dewar rotating speeds

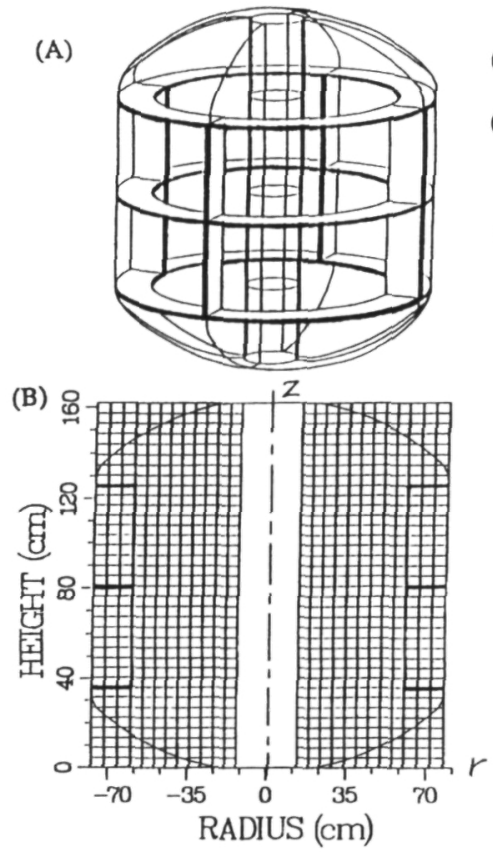

(A) Three Dimensional Profile of Baffle Boards

(B) Grid Points in Radial Axial Direction With Baffle Boards

(C) Grid Points in Radial - Circumferential Direction With Baffle Boards

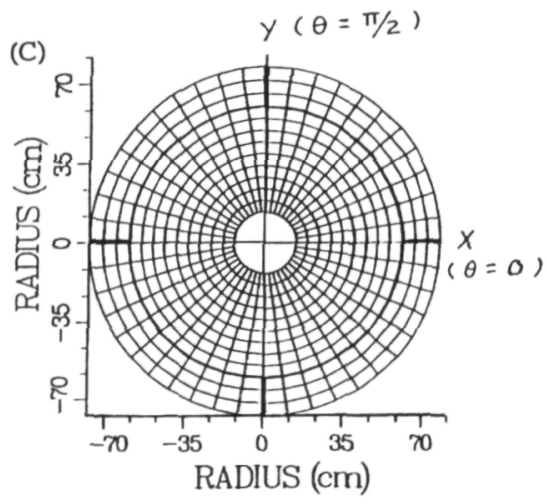

Figure 2 (a) Three-dimensional configuration of container with baffle; (b) distribution of grid points in radial-axial plane; and (c) in the radial-circumferential plane 
(a) $\mathrm{t}=225 \mathrm{~s}, \omega=0.1 \mathrm{rpm}$

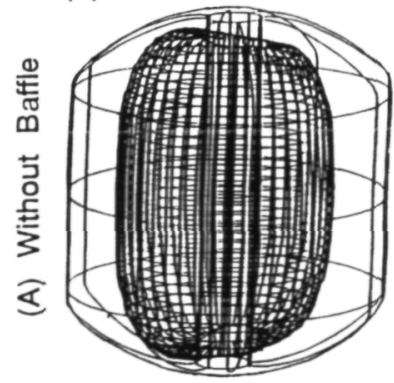

(a) $\mathrm{t}=225 \mathrm{~s}, \omega=0.1 \mathrm{rpm}$

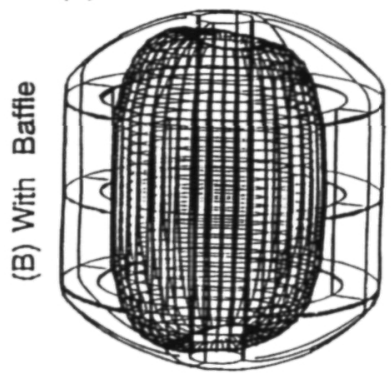

(b) $\mathrm{t}=91.8 \mathrm{~s}, \omega=0.2 \mathrm{rpm}$

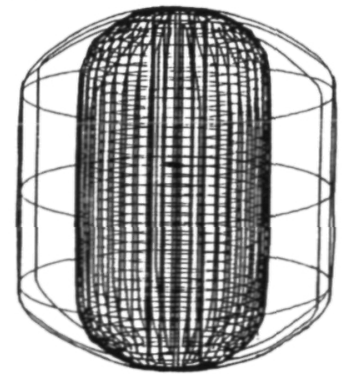

(b) $\mathrm{t}=91.8 \mathrm{~s}, \omega=0.2 \mathrm{rpm}$

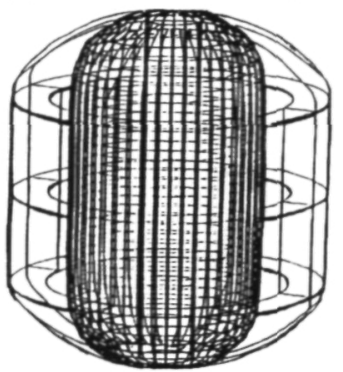

(c) $\mathrm{t}=473 \mathrm{~s}, \omega=0.3 \mathrm{rpm}$

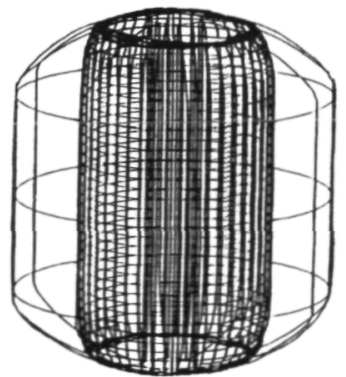

(c) $\mathrm{t}=473 \mathrm{~s}, \omega=0.3 \mathrm{rpm}$

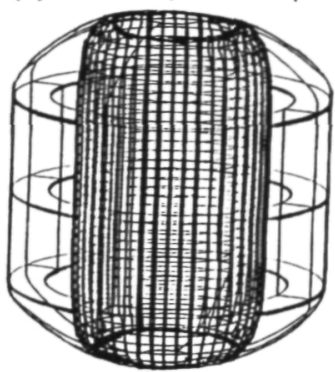

Figure 3 Three-dimensional bubble deformation for Dewar with rotating speeds of $0.1,0.2$ and 0.3 rev $\mathrm{min}^{-1}$ in response to a lateral impulse for container without and with baffle
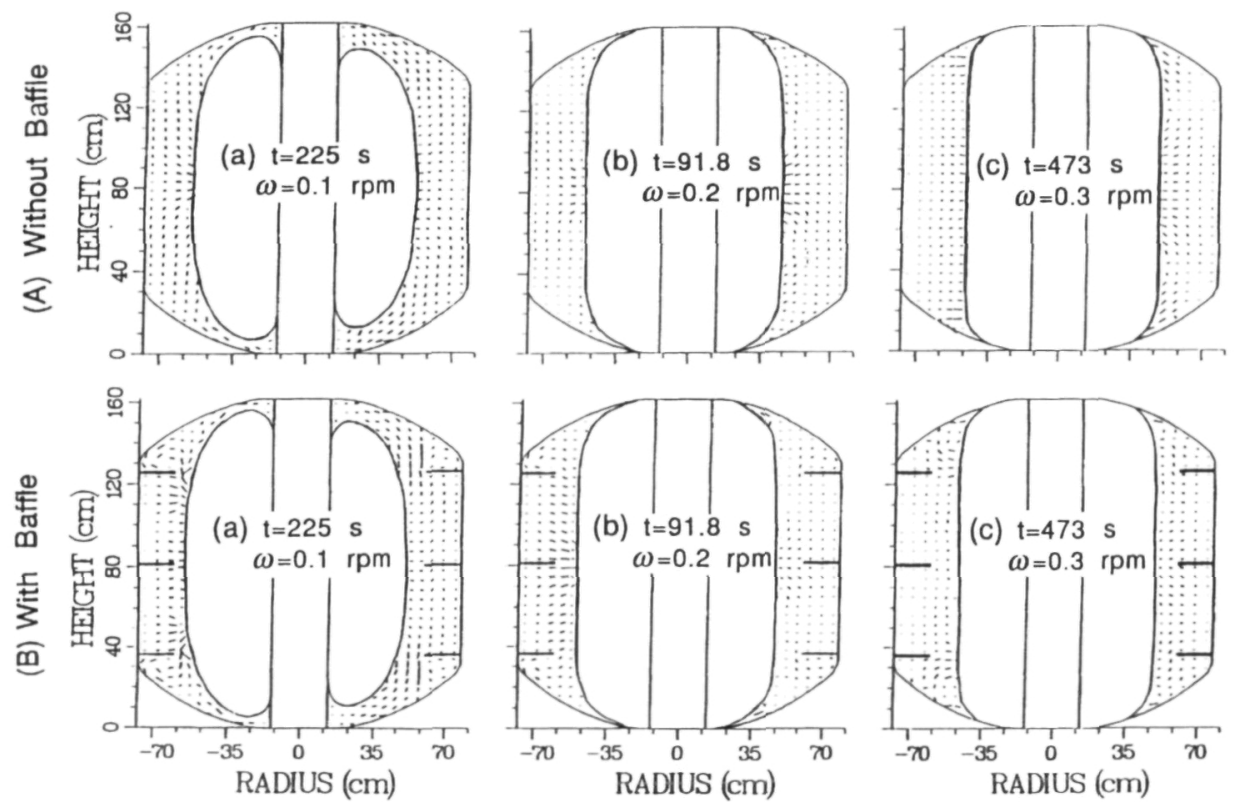

Figure 4 Bubble deformation for Dewar with rotating speeds of $0.1,0.2$ and 0.3 rev $\min ^{-1}$ in the $r-z$ plane at $\theta=0^{\circ}$ and $180^{\circ}$, in response to a lateral impulse for container without and with baffle

of $0.1,0.2$ and $0.3 \mathrm{rev} \mathrm{min}^{-1}$, respectively, without a baffle in the vertical $r-z$ plane at $\theta=0^{\circ}$ and $180^{\circ}$, in response to the same lateral impulse.

Comparison of Figures 3(A) and $4(A)$ for bubble deformations in a rotating Dewar without a baffle leads to the following conclusions: 1 , an eccentric-wheel type of rotation in bubbles demonstrates that the bubble swings around with respect to the rotating axis, and that the bubble swings from the left to the right side, as shown in Figures 3 and 4 in response to a lateral impulse; 2 , the bubble is floating in the middle of the rotating Dewar with a speed of $0.1 \mathrm{rev} \mathrm{min}^{-1}-$ it elongates vertically and contacts the upper and lower walls of the Dewar as the rotating speeds increase to 0.2 rev $\min ^{-1}$ and above; 3 , the area of bubble contacting the upper and lower walls of the Dewar increases as the rotating speed increases to $0.2 \mathrm{rev} \mathrm{min}^{-1}$ and above; 4 , the maximum cross-sectional area of the bubble decreases as the rotating speed increases; 5, the maximum radius of baffle swelling during the asymmetric oscillations decreases as the rotating speed increases; and 6 , increasing the rotating speed, which results in an increase of centrifugal force, contributes to the stability of the bubble in response to the lateral impulse.

In this study, the time evolution of the liquid-vapour 
interface oscillations for the container with a baffle due to sloshing dynamics driven by the same impulsive acceleration as that acting on the Dewar without a baffle has been investigated. Figures $3(B) a, b$ and $c$ show three-dimensional bubble deformations with Dewar rotating speeds of $0.1,0.2$ and $0.3 \mathrm{rev} \mathrm{min}^{-1}$, respectively, with baffles, in response to a lateral impulse. Figures $4(B) a, b$ and $c$ show the corresponding bubble deformations accompanied by flow profiles with Dewar rotating speeds of $0.1,0.2$ and 0.3 rev $\min ^{-1}$, respectively, with baffles in the vertical $r-z$ plane at $\theta=0^{\circ}$ and $180^{\circ}$, in response to the same lateral impulse. Comparison of Figures 3 and 4 for bubble deformations in the rotating Dewar with and without a baffle in response to a lateral impulse leads to the following conclusions: 1, the conclusions drawn for the bubble oscillations shown in Figures $3(A)$ and $4(A)$ for a rotating Dewar without a baffle are also applicable for the Dewar with a baffle; 2, bubble oscillations in the rotating Dewar with a baffle demonstrate smaller amplitudes of fluctuation than those for the Dewar without a baffle; and 3, a different flow profile around the rotating bubble is seen for the Dewar with a baffle, in which the flow around the bubble circumvents the baffle (which is not available for flow without a baffle).

\section{Sloshing reaction forces and torques exerted on the Dewar in response to an impulse}

In this study, the characteristics of the slosh reaction forces and torque fluctuations exerted on the Dewar in response to a lateral impulse are investigated. The mathematical formulation of the fluctuations of the slosh reaction forces and torques exerted on the Dewar are illustrated precisely in the paper given by Hung et al. ${ }^{27}$. With reference to the previous section for sloshing dynamics driven by an impulse and mathematical formulations ${ }^{27}$, one can calculate the slosh reaction force and the associated moments acting on the Dewar.

Figure 5 shows the computed time variation of the fluctuations of the slosh reaction force, with solid and broken lines along the $x$ - and $y$-axes, respectively, for the rotating Dewar without a baffle, exerted on the Dewar in response to the impulse. Figures $5 a, b$ and $c$ illustrate the time evolution of the fluctuations of the slosh reaction forces exerted on the container with rotating speeds of $0.1,0.2$ and $0.3 \mathrm{rev} \mathrm{min}^{-1}$, respectively, for a Dewar without a baffle. As the impulse was applied laterally along the $x$-axis with container-bound co-ordinates, the slosh reaction forces acting on the container were in the radial direction along the $x$ - and $y$-axes, and there is no force in the $z$-axis.

Table 1 shows the characteristics of the slosh reaction forces exerted on the Dewar without a baffle in response to a lateral impulse, based on Figure 5. The following conclusions can be drawn from Figure 5 and Table 1: 1, fluid flows with higher Dewar rotating speeds imply that the rotating fluid is associated with a higher energy system; 2, the maximum and minimum slosh reaction forces acting on the rotating Dewar in response to the lateral impulse increase as the Dewar rotating speed increases; and 3, the Dewar with a higher rotating speed requires a longer time period to dissipate the sloshing perturbations because of the association of the sloshing wave with higher energy for higher rotating speeds.

There are some peculiar differences shown between the
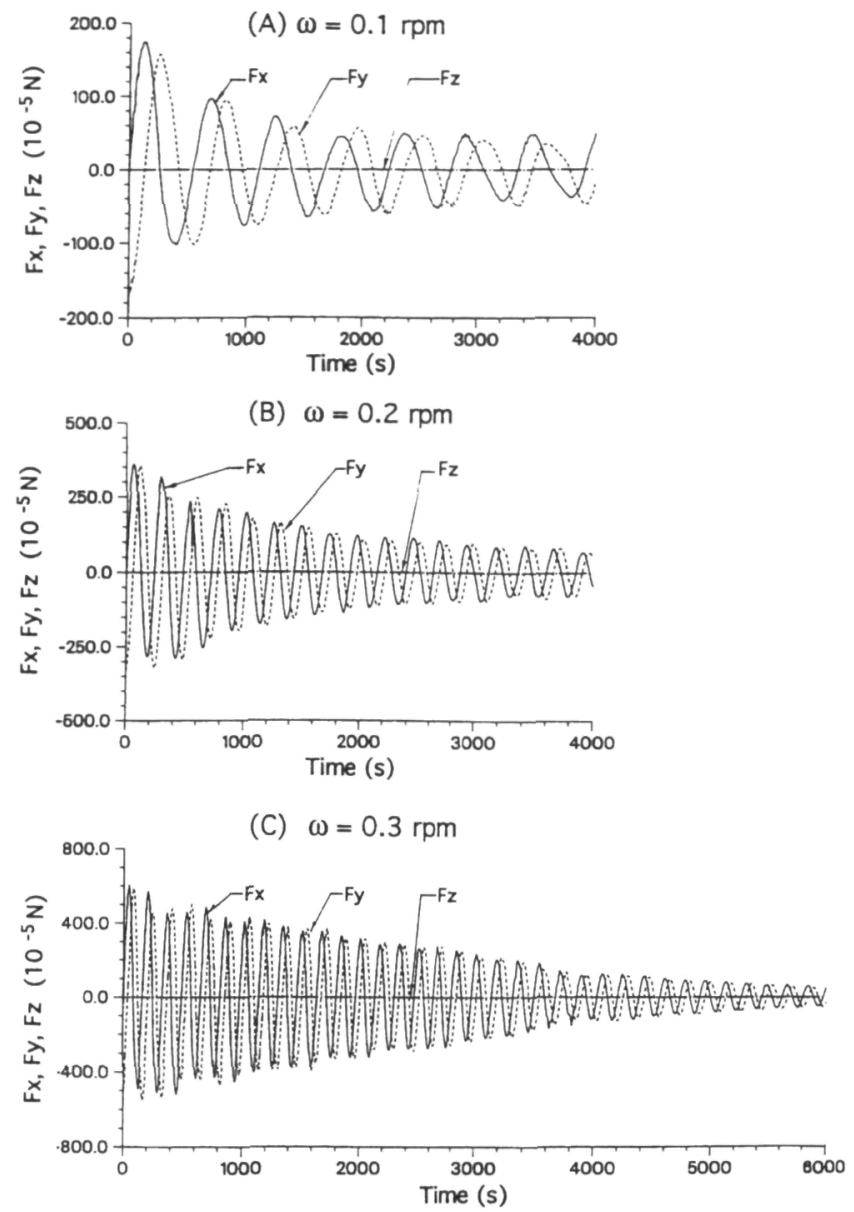

Figure 5 Time sequence evolution of fluid feedback force fluctuations exerted on Dewar with rotating speeds of $0.1,0.2$ and $0.3 \mathrm{rev} \mathrm{min}^{-1}$ along the $x-, y$ - and $z$-axes in response to a lateral impulse for container without baffle

results of the plots illustrated in Figure 5 and the data shown in Table 2. In Figure 5, the maximum absolute values of slosh reaction forces exerted on the container without a baffle are of the order of a few hundred dynes. On the other hand, in the data, the maximum absolute value of slosh reaction forces exerted on the container without a baffle are of the order of values greater than ten newtons ( $10^{6}$ dyne). From Table 2 we can draw the following results: 1 , for time $t<100 \mathrm{~ms}$, the slosh reaction forces in response to a lateral impulse introduce a large amplitude force greater than $10^{6}$ dyne, which is exerted on the Dewar at the moment the impulse is applied; 2, the magnitude of these instant slosh reaction forces decreases as the Dewar rotating speeds increase, because higher centrifugal forces contribute to the stability of the fluid system; and 3, these large amplitude slosh reaction forces damp out quickly at time $t>1 \mathrm{~s}$.

To understand this instant excitation and dissipation of slosh reaction forces in the initial stage, the time average of the impulse force in response from the cryogenic fluid to the container is computed. The time average impulse force for an impulse from time $t=0$ to $t_{k}$ is

$I_{d, i}^{k}=\frac{\int_{0}^{t_{k}} F_{i} \mathrm{~d} t}{\int_{0}^{t_{k}} \mathrm{~d} t}($ dyne $)$ 
Table 1 Characteristics of slosh reaction force for times $>1 \mathrm{~s}$ after end of lateral impulse

\begin{tabular}{|c|c|c|c|c|c|c|}
\hline \multirow{2}{*}{$\begin{array}{l}\text { Rotating speed } \\
\left(\text { rev } \min ^{-1}\right)\end{array}$} & \multirow[b]{2}{*}{ Baffle } & \multicolumn{2}{|c|}{$\begin{array}{l}\text { Maximum (minimum) slosh } \\
\text { reaction forces }\end{array}$} & \multirow{2}{*}{$\begin{array}{l}\text { Time required } \\
\text { to dissipate } \\
\text { perturbation } \\
\text { (s) }\end{array}$} & \multirow{2}{*}{$\begin{array}{l}\text { Wave period } \\
\text { excited (s) }\end{array}$} & \multirow{2}{*}{$\begin{array}{l}\text { Force reduction } \\
\text { due to baffle } \\
(\%)\end{array}$} \\
\hline & & $F_{x}\left(\times 10^{-5} \mathrm{~N}\right)$ & $F_{y}\left(\times 10^{-5} \mathrm{~N}\right)$ & & & \\
\hline \multirow[t]{2}{*}{0.1} & Without & 173 & -178 & 1300 & 571 & 21 \\
\hline & With & 128 & -152 & 600 & 667 & \\
\hline \multirow[t]{2}{*}{0.2} & Without & 360 & -372 & 2200 & 235 & 14 \\
\hline & With & 310 & -320 & 1800 & 267 & \\
\hline \multirow[t]{2}{*}{0.3} & Without & 602 & -612 & 6000 & 167 & 11 \\
\hline & With & 535 & -550 & 2600 & 182 & \\
\hline
\end{tabular}

Table 2 Maximum and minimum slosh reaction forces for less than initial $100 \mathrm{~ms}$

\begin{tabular}{|c|c|c|c|c|c|c|c|c|c|}
\hline \multirow[b]{3}{*}{ Baffle } & \multirow[b]{3}{*}{$\begin{array}{l}\text { Rotating } \\
\text { speed } \\
\text { (rev } \min ^{-1} \text { ) }\end{array}$} & \multicolumn{4}{|c|}{ Maximum slosh reaction forces } & \multicolumn{4}{|c|}{ Minimum slosh reaction forces } \\
\hline & & \multicolumn{2}{|c|}{$X$-component } & \multicolumn{2}{|c|}{$Y$-component } & \multicolumn{2}{|c|}{$X$-component } & \multicolumn{2}{|c|}{$Y$-component } \\
\hline & & $\begin{array}{l}\text { Time } \\
\left(\times 10^{-3} \mathrm{~s}\right)\end{array}$ & $\begin{array}{l}F_{x} \\
\left(\times 10^{-1} N\right)\end{array}$ & $\begin{array}{l}\text { Time } \\
\left(\times 10^{-3} \mathrm{~s}\right)\end{array}$ & $\begin{array}{l}F_{y} \\
\left(\times 10^{-1} \mathrm{~N}\right)\end{array}$ & $\begin{array}{l}\text { Time } \\
\left(\times 10^{-3} \mathrm{~s}\right)\end{array}$ & $\begin{array}{l}F_{x} \\
\left(\times 10^{-1} N\right)\end{array}$ & $\begin{array}{l}\text { Time } \\
\left(\times 10^{-3} \mathrm{~s}\right)\end{array}$ & $\begin{array}{l}F_{y} \\
\left(\times 10^{-1} \mathrm{~N}\right)\end{array}$ \\
\hline \multirow[t]{3}{*}{ Without } & 0.1 & 2.0 & 146.0 & 1.0 & 24.4 & 16.0 & -3.81 & 4.0 & -3.35 \\
\hline & 0.2 & 8.0 & 145.7 & 18.0 & 0.08 & 11.0 & -1.94 & 16.0 & -2.26 \\
\hline & 0.3 & 4.3 & 143.8 & 35.0 & 0.06 & 19.0 & -1.63 & 1.0 & -1.47 \\
\hline \multirow[t]{3}{*}{ With } & 0.1 & 1.0 & 162.2 & 1.0 & 91.0 & 21.0 & -17.72 & 25.0 & -3.41 \\
\hline & 0.2 & 4.0 & 149.7 & 6.0 & 1.42 & 17.0 & -2.57 & 7.0 & -0.64 \\
\hline & 0.3 & 1.0 & 146.5 & 1.0 & 0.34 & 15.0 & -1.14 & 2.0 & -0.09 \\
\hline
\end{tabular}

During the initial time period of $1 \mathrm{~s}$, the major contributions are the component along the $x$-direction, and the contributions from the components in the rest of the directions are negligibly small. Table 3 shows the time-averaged impulsive forces acting on the Dewar in response to a lateral impulse at time $t=0.01,0.02,0.59,100,1000$ and $4000 \mathrm{~s}$. This leads to the following conclusions for the Dewar without a baffle: 1 , in the initial stage with time $t<1 \mathrm{~s}$, a great amplitude of slosh reaction force is immediately induced in response to a lateral impulse; 2 , the amplitude of slosh reaction force acting on the rotating Dewar increases as the Dewar rotating speed increases; 3 , in the later stage of development, for time $t>1 \mathrm{~s}$, a dramatic damping effect causes the time-averaged impulsive force to reduce to the order of 1000 dyne, and then to the order of 100 dyne for time $t>100 \mathrm{~s}$; and 4, it takes a longer time to damp out the slosh reaction force fluctuations associated with Dewars with higher rotating speeds because of the association with higher wave energy.

The power spectrum of the slosh reaction force fluctu- ations for a Dewar without a baffle in response to a lateral impulse is analysed through fast Fourier analysis of data obtained from Figure 5. Figure $6 a$ shows the power spectrum of the slosh reaction force fluctuations for a Dewar without a baffle for rotating speeds of $0.1-0.3 \mathrm{rev} \mathrm{min}^{-1}$, in response to a lateral impulse. With reference to Figure 6 , Table 1 lists the values of wave period of slosh reaction force fluctuations excited by the lateral impulse. This leads to the following results based on Figure $6 a$ and Table I: 1 , wave periods of 571, 235 and $167 \mathrm{~s}$ excited for slosh reaction force fluctuations corresponding to Dewar rotating speeds of $0.1,0.2$ and $0.3 \mathrm{rev} \mathrm{min}^{-1}$, respectively, have been obtained for a Dewar without a baffle; 2, Dewars with higher rotating speeds are responsible for the excitation of sloshing waves with shorter wave periods (higher frequency modes) than wave modes with longer wave periods (lower frequency modes) for Dewars with lower rotating speeds; 3 , the higher frequency sloshing modes associated with Dewars of a higher rotating speed (higher centrifugal force) are responsible for the excitation of sloshing modes

Table 3 Time-averaged impulsive forces acting on Dewar

\begin{tabular}{|c|c|c|c|c|c|c|c|}
\hline \multirow[b]{3}{*}{ Baffle } & \multirow{3}{*}{$\begin{array}{l}\text { Rotating } \\
\text { speed } \\
\text { (rev } \text { min }^{-1} \text { ) }\end{array}$} & \multicolumn{6}{|c|}{ Time-averaged impulsive forces acting on Dewar $\left(\times 10^{-5} \mathrm{~N}\right)$} \\
\hline & & \multicolumn{6}{|c|}{ Time period of impulsive force activation (s) } \\
\hline & & 0.01 & 0.02 & 0.59 & 100 & 1000 & 4000 \\
\hline \multirow[t]{3}{*}{ Without } & 0.1 & $1252 \times 10^{3}$ & $669 \times 10^{3}$ & $23 \times 10^{3}$ & 295 & 134 & 71.9 \\
\hline & 0.2 & $1320 \times 10^{3}$ & $721 \times 10^{3}$ & $25 \times 10^{3}$ & 493 & 280 & 155.2 \\
\hline & 0.3 & $1352 \times 10^{3}$ & $730 \times 10^{3}$ & $27 \times 10^{3}$ & 706 & 493 & 291.3 \\
\hline \multirow[t]{3}{*}{ With } & 0.1 & $1329 \times 10^{3}$ & $728 \times 10^{3}$ & $27 \times 10^{3}$ & 278 & 101 & 40.0 \\
\hline & 0.2 & $1342 \times 10^{3}$ & $740 \times 10^{3}$ & $30 \times 10^{3}$ & 439 & 212 & 93.0 \\
\hline & 0.3 & $1380 \times 10^{3}$ & $758 \times 10^{3}$ & $35 \times 10^{3}$ & 665 & 323 & 124.0 \\
\hline
\end{tabular}


(a) $\omega=0.1 \mathrm{rpm}$

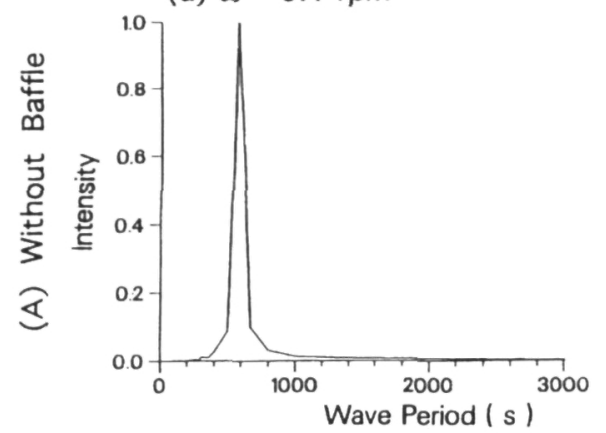

(a) $\omega=0.1 \mathrm{rpm}$

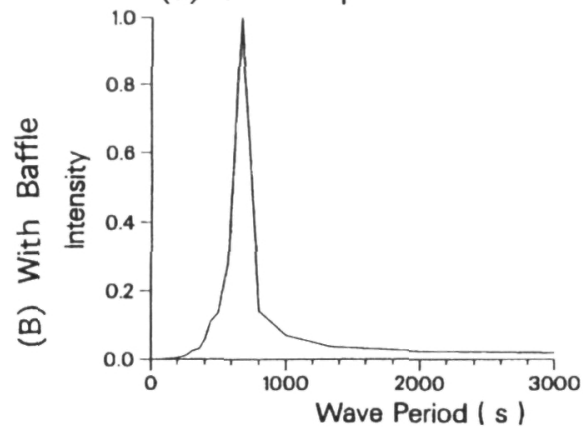

(b) $\omega=0.2 \mathrm{rpm}$

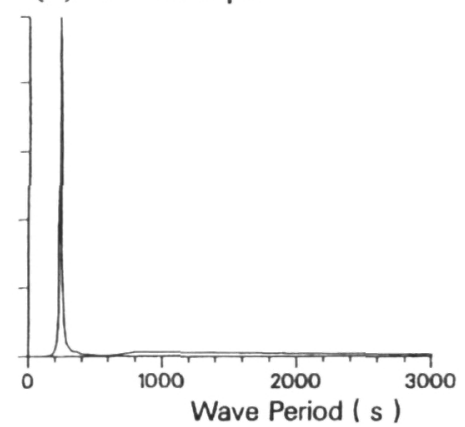

(b) $\omega=0.2 \mathrm{rpm}$

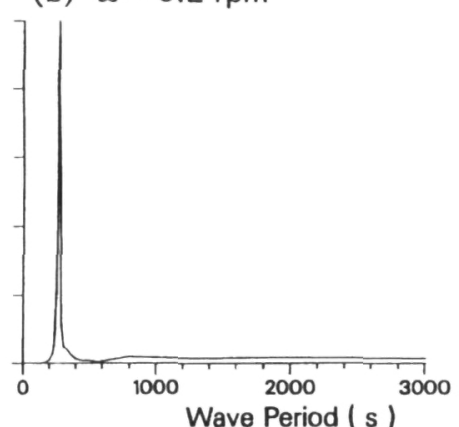

(c) $\omega=0.3 \mathrm{rpm}$

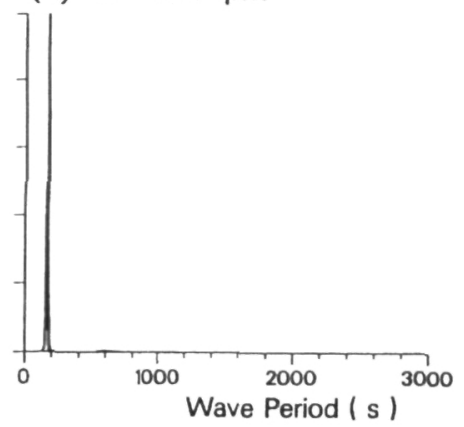

(c) $\omega=0.3 \mathrm{rpm}$

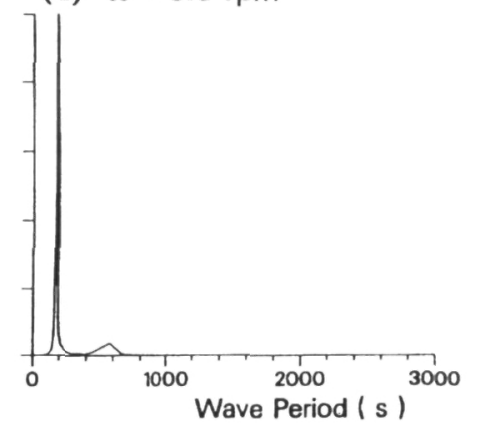

Figure 6 Power spectra of fluid feedback force fluctuations exerted on Dewar with rotating speeds of $0.1,0.2$ and 0.3 rev min ${ }^{-1}$ in response to lateral impulse for container without and with baffle

with a higher wave energy, which require a longer time period to damp out the waves than those excited by a Dewar with lower rotating speed.

As for the slosh wave induced fluid torque exerted on the Dewar without a baffle in response to the lateral impulse, calculation shows that it is negligibly small because all the slosh reaction forces are pointing towards the Dewar mass centre.

In this study, the characteristics of the slosh reaction forces and the torque fluctuations acting on the Dewar with a baffle in response to the lateral impulse are also investigated. Figure 7 illustrates the time evolution of the fluctuations of slosh reaction forces exerted on the Dewar with rotating speeds of $0.1-0.3 \mathrm{rev} \mathrm{min}^{-1}$ for the Dewar with a baffle. Table $l$ also shows the characteristics of the slosh reaction forces exerted on the Dewar with a baffle in response to a lateral impulse based on Figure 7. Comparison of Figures 5 and 7 and Table 1 for slosh reaction forces exerted on the Dewar with and without a baffle in response to a lateral impulse leads to the following conclusions: 1, the conclusions for the slosh reaction force fluctuations drawn for the Dewar without a baffle are basically applicable to the Dewar with a baffle; 2 , comparison of both the maximum and minimum slosh reaction forces exerted on a container for the Dewar with and without a baffle shows that the forces are smaller for the Dewar with baffle than for the Dewar without a baffle; 3, the Dewar with a baffle provides a very effective damping mechanism of viscosity dissipation for fluctuations between liquid-solid and vapour-solid interfaces and ends up with a reduction of slosh reaction forces in the Dewar with and without a baffle in the range of 21,14 and $11 \%$ reduction each for Dewars with rotating speeds of $0.1,0.2$ and $0.3 \mathrm{rev} \mathrm{min}^{-1}$, respectively; and 4, the times required to dissipate the slosh reaction force fluctuations show that a longer period is required to damp out force fluctuations for the Dewar without a baffle than for the Dewar with a baffle.

Comparison of the maximum absolute values of slosh reaction forces exerted on the Dewar with a baffle and the results of the plots illustrated in Figure 7 and the data shown in Table 2 is also made. Similar to the results shown in Figure 5 and Table 2 for the Dewar without baffle, a large amplitude of the order of values greater than $10^{6}$ dynes of slosh reaction forces is also instantly introduced in response to the lateral impulse for the Dewar with a baffle. Figures 5 and 7 and Table 2 for the Dewar with and without a baffle lead to the following conclusions: 1, the conclusions drawn for the slosh reaction force fluctuations for the Dewar without a baffle are applicable to that for the Dewar with a baffle; 2, comparison of the Dewar with and without a baffle shows that the magnitudes of these instantly induced slosh reaction forces exerted on the Dewar in response to a lateral impulse during the period of time $t<100 \mathrm{~ms}$ are about the same for the Dewars with and without a baffle.

To understand this instant excitation and dissipation of slosh reaction forces in the initial stage for the Dewar with a baffle during the period of time $t<100 \mathrm{~ms}$, the accumulative impulse and the time-average of the impulse force in response from the cryogenic fluid to the Dewar are also computed. Table 3 shows the time-averaged impulsive forces acting on the Dewar with and without a baffle in response to a lateral impulse. We can now draw the following conclusions: 1, the conclusions drawn for the initial stage excitation of the slosh reaction force obtained for the Dewar without a baffle are also applicable for the Dewar with a baffle; 2 , the magnitude of the time-averaged fluid impulsive forces acting on the Dewar with a baffle are about the same order of magnitude as that for the Dewar without a baffle during the time period of $t<100 \mathrm{~ms}$; and 

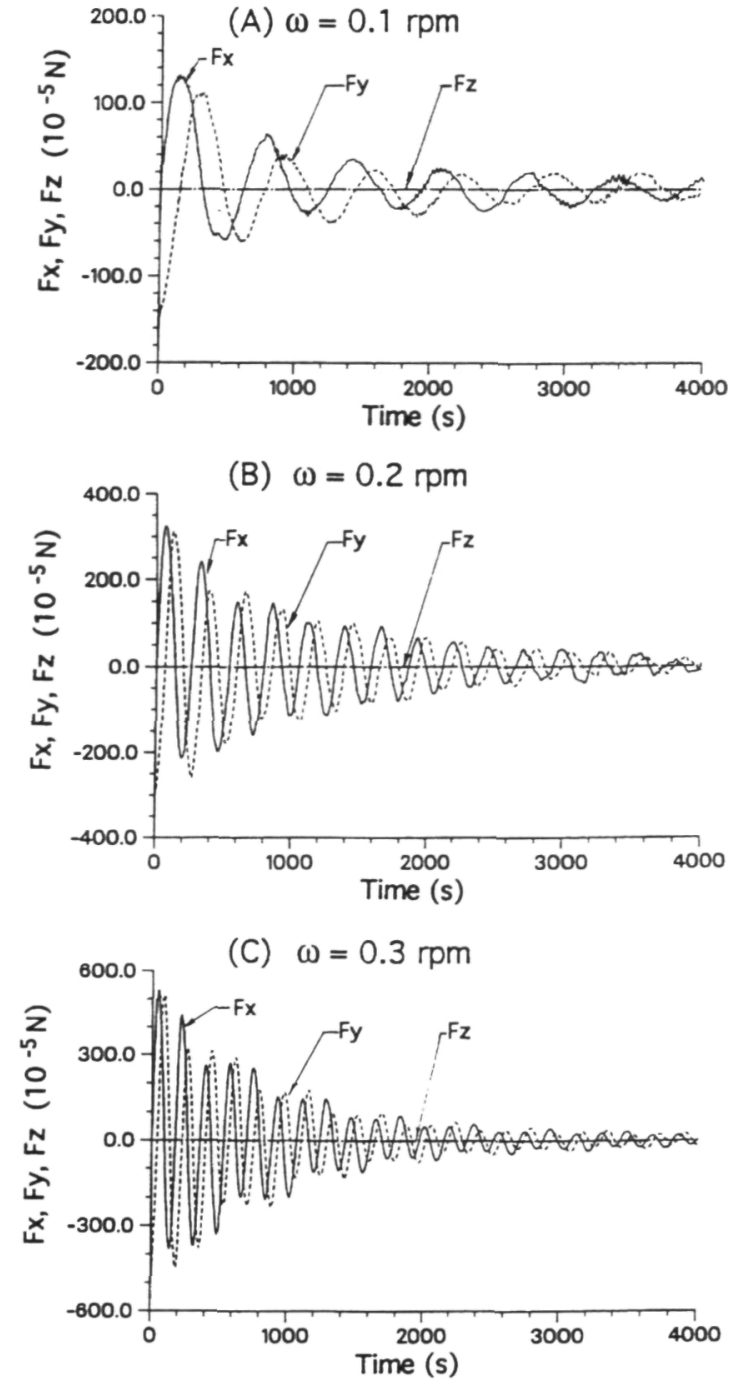

Figure 7 Time sequence evolution of fluid feedback force fluctuations on Dewar with rotating speeds of $0.1,0.2$ and 0.3 rev min $^{-1}$ in response to lateral impulse for container with baffle

3 , the greater damping effect contributed by the baffle due to viscosity dissipation for fluctuations between the liquidsolid and vapour-solid interfaces means that the magnitude of the time-averaged fluid impulsive forces acting on the Dewar with a baffle is smaller than that for the Dewar without a baffle during the time period $t>100 \mathrm{~ms}$.

The power spectrum of the slosh reaction force fluctuations for the Dewar with a baffle in response to a lateral impulse is also analysed via fast Fourier analysis of data obtained from Figure 7. Figure 6 shows the power spectrum of the slosh reaction force fluctuations for the Dewar with and without a baffle for rotating speeds of $0.1-$ $0.3 \mathrm{rev} \mathrm{min}^{-1}$, in response to a lateral impulse. With reference to Figure 6, Table 1 lists the values of wave period of slosh reaction force fluctuations excited by the lateral impulse. We can now conclude the following results for the Dewar with and without a baffle, based on Table 1 and Figure 6: 1, the conclusions drawn for wave excitations associated with the slosh reaction force fluctuations for the Dewar without a baffle are also applicable for the Dewar with a baffle; 2 , wave periods of 571,235 and 167 s excited for the Dewar without baffle and of 667, 267 and $182 \mathrm{~s}$ excited for the Dewar with a baffle have been obtained, and these values are associated with slosh reaction force fluctuations corresponding to Dewar rotating speeds of 0.1 , 0.2 and $0.3 \mathrm{rev} \mathrm{min}^{-1}$, respectively; 3 , for wave modes excited by slosh reaction force fluctuations, the Dewar with lower rotating speeds and the Dewar with a baffle are responsible for the excitation of longer period (lower frequency modes) wave modes than the wave modes with shorter periods (higher frequency modes) for the Dewar with a higher rotating speed and the Dewar without a baffle; 4 , the lower frequency sloshing modes associated with a Dewar of lower rotating speed and a Dewar with a baffle indicate that the Dewar of lower rotating speed (lower centrifugal force) together with the Dewar with a baffle are responsible for excitation of sloshing modes with lower wave energy, which require a shorter time period to damp out the waves than those excited by a Dewar with a higher rotating speed and a Dewar without a baffle; and 5, results show that the Dewar with a baffle is able to reduce the slosh reaction force fluctuations for a Dewar with rotating speeds of $0.1,0.2$ and $0.3 \mathrm{rev} \mathrm{min}^{-1}$ by 21,14 and $11 \%$, respectively, compared to a Dewar without a baffle.

\section{Discussion and conclusions}

The sloshing dynamics of fluid system disturbances in response to a lateral impulse due to the activation of guidance and/or attitude controls for spacecraft operation ${ }^{5.27}$ in microgravity have been investigated. In this study, the effect of surface tension on a partially filled rotating Dewar (liquid helium and helium vapour) with various rotating speeds activated by a time-dependent impulse in the lateral $x$-direction with and without a baffle has been considered. The study is based on the computation of three-dimensional non-inertial frame Navier-Stokes equations subject to the initial and boundary conditions $\mathrm{s}^{22,23}$. The initial conditions for the liquid-vapour interface profiles were adopted from the steady state formulations for a rotating Dewar developed by Hung and Leslie ${ }^{20}$ and by Hung et al. ${ }^{7}$.

In response to a lateral impulse, the sloshing dynamics are responsible for the excitation of sloshing waves of higher frequency modes for Dewars with higher rotating speeds. On the other hand, sloshing dynamics also account for the initiation of sloshing waves of higher frequency modes for Dewars without a baffle. These higher frequency modes are inherently associated with waves of higher energy, which requires a longer time period to damp out the fluctuations.

Because of the peculiar physical properties of liquid helium ${ }^{15-20}$, extremely low values of surface tension and viscosity coefficient of the materials make the oscillations of the bubbles and sloshing dynamics acting on the container in response to the impulse continue for a long period of time and gradually decay exponentially. This paper also discusses how a baffle installed in the container can provide an effective mechanism to damp out these fluctuations. In this research, it was demonstrated how the amplitude of the cryogenic helium sloshing dynamics disturbances grow and decay in response to a lateral impulse for a Dewar with various rotating speeds in microgravity. A full understanding of the time history of the growth and decay of the disturbances is important for the design of guidance and attitude control mechanisms for spacecraft utilizing cryogenic liquid systems $s^{5,27}$. 


\section{Acknowledgements}

The authors appreciate the support received from National Aeronautics and Space Administration through NASA grant NAG8-938 and NASA contract NAS839609/Delivery Order No. 103. They would like to express their gratitude to Gerry Nurre and Dana Billings of NASA/Marshall Space Flight Center for the stimulating discussions during the course of the present study.

\section{References}

1 Avuduyesky, V.S. Scientific Foundations of Space Manufacturing MIR, Moscow, Russia (1984)

2 Forward, R.L. Phys Rev Series A (1982) 26735

3 Misner, C.W., Thorne, K.S. and Wheeler, J.A. Gravitation W.H. Freeman Co., San Francisco, CA, USA (1973)

4 Kamotani, Y., Prasad, A. and Oastrach, S. AIAA Journal (1981) 19511

5 Hung, R.J., Lee, C.C. and Leslie, F.W. $J$ Guidance, Control and Dynamics (1992) 15817

6 Hung, R.J., Lee, C.C. and Leslie, F.W. J Fluids and Structures (1992) 6493

7 Hung, R.J., Tsao, Y.D., Hong, B.B. and Leslie, F.W. $J$ Spacecraft and Rockets (1989) 26167

8 Leslie, F.W. J Fluid Mechanics (1985) 161269

9 Mason, P., Collins, D., Petrac, D., Yang, L. et al. Proc ICEC 7 IPC Science and Technology Press, Guildford, UK (1978)

10 Donnelly, R.J. Superfluid turbulence. Scientific American (Nov 1988) 100
11 Van Sciver, S.W. Helium Cryogenics Plenum Press, New York, USA (1986)

12 Donnelly, R.J. Quantized Vertices in Helium II Cambridge University Press, Cambridge, UK (1991)

13 Hung, R.J., Pan, H.L. and Long, Y.T. Cryogenics (1994) 34641

14 Wilks, H. The Properties of Liquid and Solid Helium Clarendon Press, Oxford, UK (1967)

15 Hoare, F.E., Jackson, L.C. and Kurti, N. Experimental Cryogenics: Liquid Helium II Butterworths, London, UK (1961)

16 Hung, R.J. and Pan, H.L. Trans Jpn Soc Aeronautical and Space Sciences (1993) 36153

17 Hung, R.J., Pan, H.L. and Leslie, F.W. Fluid Dynamics Research (1994) 1429

18 Hung, R.J., Pan, H.L. and Leslie, F.W. Z Fluwiss Weltraumforsch (1994) 18195

19 Hung, R.J. and Pan, H.L. Acta Mechanica Sinica (1993) 9298

20 Hung, R.J. and Leslie, F.W. J Spacecraft and Rockets (1988) 2570

21 Harlow, F.H. and Welch, F.E. Physics of Fluids (1965) 82182

22 Hirt, C.W. and Nichols, B.D. J Computational Physics (1981) 39 201

23 Salvadori, M.G. and Baron, M.L. Numerical Methods in Engineering, Prentice-Hall, Inc., Englewood Cliffs, NJ, USA (1961)

24 Hageman, L.A. and Young, D.M. Applied Iterative Methods Academic Press, New York, USA (1981)

25 Young, D. Trans Am Mathematical Soc (1954) 7692

26 Patanker, S.V. Numerical Heat Transfer and Fluid Flow Hemisphere-McGraw Hill, New York, USA (1980) 197

27 Hung, R.J., Lee, C.C. and Leslie, F.W. Trans Jpn Soc Aeronautical and Space Sciences (1993) 35187

28 Hung, R.J. and Pan, H.L. Fluid force activated spacecraft dynamics driven by gravity gradient and jitter accelerations $J$ Guidance, Control and Dynamics (1995) 18 in press 